\title{
Directed long-range transport of a nearly pure component atom clusters by the electromigration of a binary surface alloy
}

\author{
Mikhail Khenner*1,2 \\ ${ }^{1}$ Department of Mathematics, Western Kentucky University, Bowling Green, KY 42101, USA \\ ${ }^{2}$ Applied Physics Institute, Western Kentucky University, Bowling Green, KY 42101, USA
}

(Dated: June 10, 2021)

\begin{abstract}
Assuming a vacancy-mediated diffusion, a continuum model for electromigration-driven transport of an embedded atom cluster across a surface terrace of a phase-separating $A_{x} B_{1-x}$ surface alloy, such as fcc $\operatorname{AgPt}(111)$, is presented. Computations show that the electron wind carries the cluster over hundreds of lattice spacings and in the set direction, while the cluster grows and its purity improves during the drift. Impacts of the current density, the diffusion anisotropy, the magnitude and sign of the ratio of the effective charges $q_{A} / q_{B}$, and the jump frequencies ratio $\Gamma_{A} / \Gamma_{B}$ on the cluster's drift speed, drift direction, purity and shape are demonstrated.
\end{abstract}

Keywords: Surface electromigration; surface alloys; vacancy-mediated diffusion; directed selfassembly; composition patterning.

\section{INTRODUCTION}

STM studies of the early 2000's [1-3] demonstrated that atoms in close-packed crystal surfaces of pure metals or alloys are highly mobile even at room temperature. For instance, Pd or In atoms incorporated into the topmost layer of $\mathrm{Cu}(001)$ substrate may frequently jump over distances larger than one lattice spacing, some as far as five lattice spacings. These jumps are enabled by a fast two-dimensional random walk of vacancies. Of course, the observed two-dimensional motion of impurity atoms reflects the diffusion of all copper atoms in the surface layer. Such vacancy-mediated diffusion of the surface atoms was compared to a giant atomic slide-puzzle [4.

Concerning this phenomenon, the general question one may be asking is this: Is it possible to channel, or bias, the random vacancy-mediated diffusion of individual surface atoms into a collective, directed transport across the surface by the application of the external field or force? In this communication, we answer positively. Using a continuum model based only on the classical diffusion and electromigration phenomenology [5-7, we show that the "electron wind" may transport, over distances of hundreds of lattice spacings and in the set direction on the surface, the compact clusters comprising of, say, predominantly B atom species. As the cluser is transported along the terrace, the persistant phase separation ensures the continuing in-flow of B atoms and the corresponding out-flow of A atoms from the cluster, until a $100 \%$ purity is achieved. The basic necessary conditions are that a phase-separating instablity of the surface alloy is activated, and that prior to the onset of the electromigration, there exists a small seed cluster with a spiked concentration of B atoms. Such seed cluster may be artificially created by a local implantation of B atoms into $\mathrm{A}_{x} \mathrm{~B}_{1-x}$ surface alloy, or it may occur naturally in the said alloy.

The model and the computations are presented in sections II and III respectively. The model extends the analysis in Ref. 8 and provides a self-consistent formulation, based on irreversible thermodynamics, of the vacancy-mediated embedded atom surface diffusion and electromigration. It is appropriate to remark here that several useful models of surface electromigration-driven nanoisland motion and instabilities were published [9 [15, however, these studies do not include alloys and the diffusing and migrating species in this case are the adatoms - thus the diffusion is by the direct exchange of lattice positions.

It is important to point out that the nature of a phase-separating instability is not highly important for the cluster transport on a surface. Any physical mechanism that enhances, in the non-explosive fashion, the initial localized phase inhomogeneity would suffice in the presented model, although the drift speed of the cluster and the (time-dependent) cluster purity will be affected by the detailed workings of the instability. In this communication it is assumed that the surface alloy is thermodynamically unstable, that is, the instability is spinodal [16, 17. This is the simplest phase-separating instability that can be manifested.

Literature on surface alloys is vast, however, the majority of studied alloys are thermodynamically stable. We came across the discussion of one fcc surface alloy system, $\operatorname{AgPt}(111)$ which is thermodynamically unstable [18]. These authors describe $\operatorname{AgPt}(111)$ surface alloy as "... a solid solution with a positive enthalpy of mixing, which leads to phase separation". They find that the growth of $\mathrm{Ag}$ on $\mathrm{Pt}(111)$ results in compact Ag clusters of the average size $10 \AA$

\footnotetext{
* Corresponding author. E-mail: mikhail.khenner@wku.edu.
} 
embedded in the topmost Pt layer. (The clusters were not observed for a period of time at constant temperature, thus it is not clear whether they grow or not.) Such incomplete mixing within the surface layer is consistent with the phase diagram for a bulk AgPt alloy, which features a wide miscibility gap up to $T \sim 1400 \mathrm{~K}$ [19]. The clusters dissolve into the topmost $\mathrm{Pt}$ layer only when the film is annealed at $T>650 \mathrm{~K}$ for about one minute. It is therefore possible that application of the electromigration to $\mathrm{AgPt}(111)$ surface alloy after $\mathrm{Ag}$ clusters were formed would result in these clusters drifting within the Pt surface layer, while simultaneously growing by phase separation. In the paper we provide theoretical and computational support for precisely this scenario. It should be understood that despite us using specific published material parameters for AgPt surface alloy for computations and estimates, our model is applicable to other surface alloys that display qualitatively similar thermodynamics and kinetics. For example, Ni-based fcc alloys, such as NiCo and NiCr also have a positive enthalpy of mixing in the bulk phase [20], thus they are the potential candidates for displaying phase separation when realized as surface alloys.

We hope that the proposed transport effect of the electric current on the surface atom clusters could be demonstrated in a real-life experiment and then harnessed to enable the localized chemical functionality on metal surfaces, for instance, allow the creation of the mobile catalytic "hot-spot".

\section{THE MODEL}

We consider a thin conductive film of a uniform composition A, whereby the impurity B atoms are mixed into the topmost surface layer, thus creating a binary surface alloy. The film's surface morphology is comprised of the atomically flat terraces of width $L$ separated by steps. The electric potential difference (voltage) $V$ is applied to the opposite edges of the terrace, which results in the electric field $\boldsymbol{E}=\left(E_{0} \cos \phi_{E}, E_{0} \sin \phi_{E}\right), E_{0}=V / L$. Here $\phi_{E}$ is the angle that the electric field vector makes with the $x$-axis of the Cartesian reference frame, see Fig. 1 . Note that the $x$ and $y$ axes are chosen along the principal diffusion axes.

Let $X_{A}$ and $X_{B}$ be the local time-dependent surface atom concentrations $\left(\left[X_{A}, X_{B}\right]=c m^{-2}\right)$, and let $\nu_{A}$ and $\nu_{B}$ be the corresponding average (and constant) concentrations, that is, the number of $\mathrm{A}$ and $\mathrm{B}$ atoms per unit area in the as-prepared surface alloy prior to the onset of a phase separation and electromigration. Then

$$
\frac{X_{A}}{\nu_{A}}+\frac{X_{B}}{\nu_{B}}=C_{A}+C_{B}=1,
$$

where $C_{A}$ and $C_{B}$ are the dimensionless local time-dependent concentrations, or the local composition fractions.

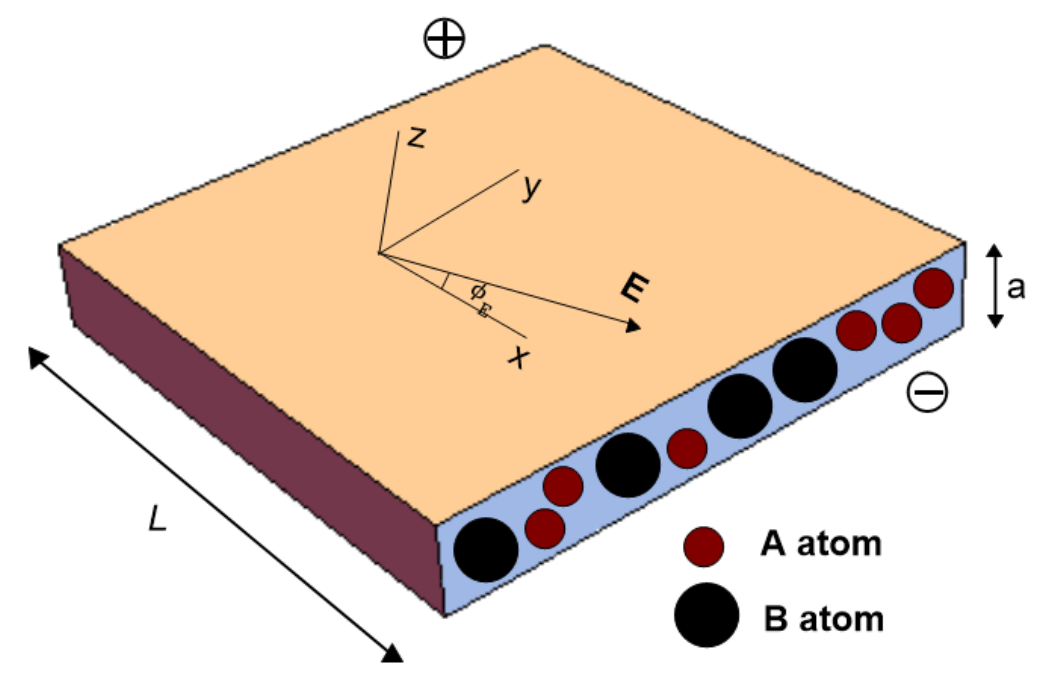

FIG. 1: Schematic representation of a single layer surface binary alloy subjected to an external electric field $\boldsymbol{E}$.

The diffusion equations read

$$
\frac{\partial X_{A}}{\partial t}=-\nabla \cdot \boldsymbol{J}_{A}, \quad \frac{\partial X_{B}}{\partial t}=-\nabla \cdot \boldsymbol{J}_{B},
$$

where $\boldsymbol{J}_{A}, \boldsymbol{J}_{B}$ are the components' fluxes measured in the laboratory reference frame, and $\boldsymbol{\nabla}=\left(\partial_{x}, \partial_{y}\right)$. We are modeling the alloyed terrace as the two-dimensional (2D) diffusion couple, where diffusion is mediated by vacancies 
(see the remarks in Sec. IV] on comparing various diffusion rates). Thus to ensure the mass conservation, according to the classical framework 21] one introduces the intrinsic (lattice) fluxes of the components, $\boldsymbol{j}_{A}$ and $\boldsymbol{j}_{B}$, that are related to the fluxes in the laboratory frame via

$$
\boldsymbol{j}_{A}=\boldsymbol{J}_{A}-\boldsymbol{v} X_{A}, \quad \boldsymbol{j}_{B}=\boldsymbol{J}_{B}-\boldsymbol{v} X_{B},
$$

where $\boldsymbol{v}(x, y, t)$ is the velocity field [7, 22. The velocity field is measured in the laboratory frame and characterizes the velocity of the 2D surface lattice relative to the bulk, non-diffusing parts of the sample away from the interdiffusion area.

The conservation laws (2) then have the form:

$$
\frac{\partial X_{A}}{\partial t}=-\boldsymbol{\nabla} \cdot \boldsymbol{j}_{A}-\boldsymbol{\nabla} \cdot\left(\boldsymbol{v} X_{A}\right), \quad \frac{\partial X_{B}}{\partial t}=-\boldsymbol{\nabla} \cdot \boldsymbol{j}_{B}-\boldsymbol{\nabla} \cdot\left(\boldsymbol{v} X_{B}\right) .
$$

Multiplying the first conservation law in Eq. (4) by $1 / \nu_{A}$, the second conservation law by $1 / \nu_{B}$, adding, and using Eq. (1) gives

$$
\boldsymbol{\nabla} \cdot \boldsymbol{v}=-\boldsymbol{\nabla} \cdot\left(\frac{\boldsymbol{j}_{A}}{\nu_{A}}+\frac{\boldsymbol{j}_{B}}{\nu_{B}}\right) .
$$

The trivial solution of this equation for $\boldsymbol{v}$ is assumed in the foregoing formulation:

$$
\boldsymbol{v}=-\frac{j_{A}}{\nu_{A}}-\frac{j_{B}}{\nu_{B}}
$$

Eq. (6) states that the lattice moves in the direction opposite to the direction of the vector sum of the lattice fluxes $\boldsymbol{j}_{A}$ and $\boldsymbol{j}_{B}$. It is expected that in practice the deviations from this rule are very small and thus in the first approximation they can be neglected $(7$, p. 198). If the deviations cannot be neglected, the analysis becomes considerably more complicated 23]. Typically, the vectors $\boldsymbol{j}_{A} / \nu_{A}$ and $\boldsymbol{j}_{B} / \nu_{B}$ (which are analogous to $V_{A} \boldsymbol{j}_{A}$ and $V_{B} \boldsymbol{j}_{B}$ for the bulk alloy, where $V_{A}$ and $V_{B}$ are the partial molar volumes [23]) point in the opposite directions and their magnitudes are nearly equal, thus the lattice velocity is very small. Yet, for bulk three-dimensional alloys it can be measured in the interdiffusion/Kirkendall experiments. In this communication, the focus is not on modeling the probable Kirkendall effect in the binary surface alloy, but on demonstrating the surface atom transport effect of the applied electric current. Thus we are not concerned with the computation of Eq. (6), and it is presented only as part of the model formulation.

With $\boldsymbol{\nabla} \cdot \boldsymbol{v}$ given by Eq. (5) and $\boldsymbol{v}$ given by Eq. (6), it is seen that the expanded conservation laws

$$
\frac{\partial X_{A}}{\partial t}=-\boldsymbol{\nabla} \cdot \boldsymbol{j}_{A}-X_{A} \boldsymbol{\nabla} \cdot \boldsymbol{v}-\boldsymbol{v} \cdot \boldsymbol{\nabla} X_{A}, \quad \frac{\partial X_{B}}{\partial t}=-\boldsymbol{\nabla} \cdot \boldsymbol{j}_{B}-X_{B} \boldsymbol{\nabla} \cdot \boldsymbol{v}-\boldsymbol{v} \cdot \boldsymbol{\nabla} X_{B}
$$

are now explicitly formulated in terms of the lattice fluxes. We now proceed to the formulation of these fluxes.

According to the classical, irreversible thermodynamics-based phenomenological model of electromigration in metal alloys [5, 6],

$$
\begin{aligned}
& \boldsymbol{j}_{\boldsymbol{A}}=-L_{A A}\left(\boldsymbol{\nabla}\left[\mu_{A}-\mu_{V}\right]-q_{A} \boldsymbol{E}\right)-L_{A B}\left(\boldsymbol{\nabla}\left[\mu_{B}-\mu_{V}\right]-q_{B} \boldsymbol{E}\right), \\
& \boldsymbol{j}_{\boldsymbol{B}}=-L_{A B}\left(\boldsymbol{\nabla}\left[\mu_{A}-\mu_{V}\right]-q_{A} \boldsymbol{E}\right)-L_{B B}\left(\boldsymbol{\nabla}\left[\mu_{B}-\mu_{V}\right]-q_{B} \boldsymbol{E}\right) .
\end{aligned}
$$

Here $L_{A A}, L_{A B}, L_{B B}$ are the kinetic transport coefficients, $\mu_{A}, \mu_{B}$ the chemical potentials of the components, $\mu_{V}$ the chemical potential of vacancies, and $q_{A}, q_{B}$ the effective surface charges of the atom species $\mathrm{A}$ and $\mathrm{B}$, respectively. The effective charges include the electrostatic and the electron-wind contributions, and are defined as

$$
q_{A}=\left(Z_{A}^{(e)}+Z_{A}^{(w)}\right) e, \quad q_{B}=\left(Z_{B}^{(e)}+Z_{B}^{(w)}\right) e,
$$

where $e<0$ is the electron charge. Note that, since the sums $Z_{i}^{(e)}+Z_{i}^{(w)}$ of the effective ion valences may be positive or negative, $q_{A}$ and $q_{B}$ also may be positive or negative. When the magnitude of the electron-wind force is much larger than the magnitude of the electrostatic force, which is typical in surface electromigration phenomena 24, 25, the positive effective charge moves in the $\boldsymbol{- E}$ direction (equivalently, in the direction of the electron flow), that is, from the negatively charged anode to the positively charged cathode. Also note that due to formulating the diffusion equations in terms of the lattice fluxes, the kinetic transport coefficients are uniquely determined and their number is reduced from four to three, in other words, the off-diagonal coefficients are equal, $L_{B A}=L_{A B}$ [5, 6]. We further assume that the vacancy sources and sinks (such as the step edges) are sufficiently effective to keep $\mu_{V}$ constant, and 
also that the binding energies V-A and V-B are equal. 43. Together, these assumptions allow to drop $\nabla \mu_{V}$ from Eqs. (8) [5].

The chemical potentials in Eq. (8) are due to alloy thermodynamics [26]:

$$
\mu_{A}=\frac{C_{B}}{\nu_{B}}\left(\frac{\partial \gamma}{\partial C_{A}}-\frac{\partial \gamma}{\partial C_{B}}\right)-\frac{\epsilon a}{\nu_{A}} \nabla^{2} C_{A}, \quad \mu_{B}=\frac{-C_{A}}{\nu_{A}}\left(\frac{\partial \gamma}{\partial C_{A}}-\frac{\partial \gamma}{\partial C_{B}}\right)-\frac{\epsilon a}{\nu_{B}} \nabla^{2} C_{B},
$$

where $\gamma$ is the free energy, $a$ the lattice spacing, and $\epsilon$ the Cahn-Hilliard gradient energy coefficient. Contributions to the chemical potentials from the compositional strain (e.g., strain that emerges because of the size mismatch of A and $\mathrm{B}$ atoms) are expected to be minor for AgPt surface alloy, since the size mismatch is not significant - the radius of $\mathrm{Ag}$ atom is $1.65 \times 10^{-8} \mathrm{~cm}$, whereas the radius of $\mathrm{Pt}$ atom is $1.77 \times 10^{-8} \mathrm{~cm}$ 27. See References 8, 28, for account of strain in a related model based on a generic diffusion setup. 44]

The free energy is chosen as [29, 30]:

$$
\gamma=\gamma_{A} C_{A}+\gamma_{B} C_{B}+k T \nu\left(C_{A} \ln C_{A}+C_{B} \ln C_{B}+H C_{A} C_{B}\right)
$$

where it is assumed $\nu_{A}=\nu_{B}=\nu, \gamma_{A}\left(\gamma_{B}\right)$ is the surface energy of atomically thin surface layer that is composed of A (B) atoms, $k T \nu$ the alloy entropy, and the dimensionless number $H=\alpha_{i n t} / k T \nu$ measures the bond strength relative to the thermal energy $k T$. Here $\alpha_{i n t}$ is the enthalpy of mixing. The terms in the parenthesis are the regular solution model. Note that when B atoms are deposited on a surface composed of A atoms, if B atoms have lower surface energy $\left(\gamma_{B}<\gamma_{A}\right)$, then they tend to be confined to the surface layer; if $\gamma_{B}>\gamma_{A}$, then B atoms tend to be dissolved in the A-bulk [29].

Apart from the constant matrix $\boldsymbol{W}$, which will be introduced shortly, the kinetic transport coefficients have the forms derived from Manning's model of diffusion in nondilute alloys [31, 32]:

$$
\begin{aligned}
L_{A A} & =\Gamma_{B} \Gamma C_{A} \frac{\lambda a^{2} \nu}{k T}\left(1-\frac{2 \Gamma C_{B}}{\psi}\right) \boldsymbol{W}, \quad L_{A B}=\Gamma_{B} \Gamma C_{A} C_{B} \frac{2 \lambda a^{2} \nu}{k T \psi} \boldsymbol{W}, \\
L_{B B} & =\Gamma_{B} C_{B} \frac{\lambda a^{2} \nu}{k T}\left(1-\frac{2 C_{A}}{\psi}\right) \boldsymbol{W},
\end{aligned}
$$

where the dimensionless $\psi$ is given by

$$
\psi=\frac{1}{2}\left(M_{0}+2\right)\left(\Gamma C_{A}+C_{B}\right)-\Gamma-1+2\left(C_{A}+\Gamma C_{B}\right)+\sqrt{\left[\frac{1}{2}\left(M_{0}+2\right)\left(\Gamma C_{A}+C_{B}\right)-\Gamma-1\right]^{2}+2 M_{0} \Gamma .}
$$

In Eqs. 12 and (13), $\Gamma=\Gamma_{A} / \Gamma_{B}, \Gamma_{A}$ and $\Gamma_{B}$ are the jump frequencies of species $\mathrm{A}$ and $\mathrm{B}$, respectively, $M_{0}=2 f_{0} /\left(1-f_{0}\right), f_{0}$ and $\lambda$ are the tracer correlation factor and the dimensionless geometric factor for fcc lattice, respectively, and $\boldsymbol{W}$ the diagonal, transversely isotropic tensor,

$$
\boldsymbol{W}=\left(\begin{array}{ll}
1 & 0 \\
0 & \Lambda
\end{array}\right)
$$

where the dimensionless $\Lambda$ measures the difference in the diffusion strength along the $x$ and $y$ axes. Specifically, if we let $D_{x}$ and $D_{y}$ be the diffusivities along the $x$ and $y$ axes, respectively, then $D_{y}=\Lambda D_{x}$. Thus the multiplication of the kinetic transport coefficients by $\boldsymbol{W}$ allows to account for the possible directional diffusional anisotropy in the xy-plane. When $\Lambda=1, \boldsymbol{W}=\boldsymbol{I}$, where $\boldsymbol{I}$ is the identity tensor, and the diffusion becomes isotropic.

Equations (1), (5)-(8), (10)-(14) constitute our dimensional model, with $\nu_{A}=\nu_{B}=\nu$ in Eq. (10). To render this model dimensionless, we chose $a$ as the length scale and $\bar{t}=k T \nu /\left(\gamma_{B} \lambda \Gamma_{B}\right)$ as the time scale. After elimination of $C_{A}$ using Eq. (1), we arrive to the final dimensionless model for the spatio-temporal dynamics of the concentration of $\mathrm{B}$ atoms:

$$
\begin{gathered}
\frac{\partial C_{B}}{\partial t}=-\boldsymbol{\nabla} \cdot\left[F_{e} \boldsymbol{\Omega}_{B}-\boldsymbol{\Sigma}_{B}+\left(\boldsymbol{\Sigma}_{\boldsymbol{A}}+\boldsymbol{\Sigma}_{\boldsymbol{B}}-F_{e} \boldsymbol{\Omega}_{A}-F_{e} \boldsymbol{\Omega}_{B}\right) C_{B}\right], \\
\boldsymbol{\Sigma}_{A}=\bar{L}_{A A} \boldsymbol{\nabla}_{\Lambda} \mu_{A}+\bar{L}_{A B} \boldsymbol{\nabla}_{\Lambda} \mu_{B}, \quad \boldsymbol{\Sigma}_{B}=\bar{L}_{A B} \boldsymbol{\nabla}_{\Lambda} \mu_{A}+\bar{L}_{B B} \boldsymbol{\nabla}_{\Lambda} \mu_{B}, \\
\boldsymbol{\Omega}_{A}=\left[Q \bar{L}_{A A}+\bar{L}_{A B}\right]\left(\begin{array}{c}
\cos \phi_{E} \\
\Lambda \sin \phi_{E}
\end{array}\right), \quad \boldsymbol{\Omega}_{B}=\left[Q \bar{L}_{A B}+\bar{L}_{B B}\right]\left(\begin{array}{c}
\cos \phi_{E} \\
\Lambda \sin \phi_{E}
\end{array}\right),
\end{gathered}
$$




$$
\begin{gathered}
\bar{L}_{A A}=\Gamma\left(1-C_{B}\right)\left(1-\frac{2 \Gamma C_{B}}{\psi}\right), \quad \bar{L}_{A B}=\frac{2}{\psi} \Gamma C_{B}\left(1-C_{B}\right), \quad \bar{L}_{B B}=C_{B}\left(1-\frac{2\left(1-C_{B}\right)}{\psi}\right), \\
\mu_{A}=-C_{B} \frac{\partial \gamma}{\partial C_{B}}+\xi \nabla^{2} C_{B}, \quad \mu_{B}=\left(1-C_{B}\right) \frac{\partial \gamma}{\partial C_{B}}-\xi \nabla^{2} C_{B}, \\
\gamma=G\left(1-C_{B}\right)+C_{B}+N\left[\left(1-C_{B}\right) \ln \left(1-C_{B}\right)+C_{B} \ln C_{B}+H C_{B}\left(1-C_{B}\right)\right], \\
\psi=\frac{1}{2}\left(M_{0}+2\right)\left(\Gamma\left(1-C_{B}\right)+C_{B}\right)-\Gamma-1+2\left(\left(1-C_{B}\right)+\Gamma C_{B}\right)+ \\
\sqrt{\left[\frac{1}{2}\left(M_{0}+2\right)\left(\Gamma\left(1-C_{B}\right)+C_{B}\right)-\Gamma-1\right]^{2}+2 M_{0} \Gamma} \\
\nabla_{\Lambda}=\left(\begin{array}{c}
\partial_{x} \\
\Lambda \partial_{y}
\end{array}\right) \cdot
\end{gathered}
$$

Here, $\boldsymbol{\nabla}_{\Lambda}$ is the anisotropic gradient operator, which reduces to the $\boldsymbol{\nabla}$ operator when diffusion is isotropic. The fluxes $\boldsymbol{\Sigma}_{A}$ and $\boldsymbol{\Sigma}_{B}$ are due to the gradients of the chemical potentials, whereas the fluxes $\boldsymbol{\Omega}_{A}$ and $\boldsymbol{\Omega}_{B}$ are the electromigration fluxes. Since the anisotropy matrix $\boldsymbol{W}$ enters the three kinetic transport coefficients in Eqs. (8), all fluxes are anisotropic either due to presence of $\nabla_{\Lambda}$, or due to presence of the column vector $\left(\begin{array}{c}\cos \phi_{E} \\ \Lambda \sin \phi_{E}\end{array}\right)$. Also, $\bar{L}_{A A}, \bar{L}_{A B}$ and $\bar{L}_{B B}$ are the dimensionless, and now isotropic, kinetic transport coefficients. [45]

Eqs. 15)-22 contain ten dimensionless parameters. Parameters $\Gamma, \Lambda, H, M_{0}$, $\phi_{E}$ were introduced above. Note,

\begin{tabular}{|c|c|c|c|}
\hline Physical parameter & Typical value & Dimensionless parameter & Typical value \\
\hline$L$ & $10^{-5} \mathrm{~cm}(100 \mathrm{~nm}) 1$ & $\Gamma$ & 2 \\
\hline$a$ & $3.3 \times 10^{-8} \mathrm{~cm} 27(0.33 \mathrm{~nm})$ & $\Lambda$ & 1 \\
\hline$\nu=a^{-2}$ & $9.18 \times 10^{14} \mathrm{~cm}^{-2}$ & $H$ & 2.27 \\
\hline$\lambda$ & $1 / 631$ & $M_{0}$ & 7.153 \\
\hline$f_{0}$ & 0.781531 & $\phi_{E}$ & $\pi$ \\
\hline$T$ & $400 \mathrm{~K} 3$ & $G$ & 1.966 \\
\hline$q_{A}, q_{B}$ & $20|e|=10^{-8}$ statC 24,25 & $N$ & 0.044 \\
\hline$V$ & $2.25 \times 10^{-2} \mathrm{~V}$ & $\xi$ & 0.31 \\
\hline$\alpha_{i n t}$ & $117 \mathrm{erg} / \mathrm{cm}^{2}$ & $Q$ & 1 \\
\hline$\gamma_{A}$ & $2.3 \times 10^{3} \mathrm{erg} / \mathrm{cm}^{2}$ & $F_{e}$ & 0.583 \\
\hline$\gamma_{B}$ & $1.17 \times 10^{3} \mathrm{erg} / \mathrm{cm}^{2} 33$ & & \\
\hline$\epsilon$ & $1.2 \times 10^{-5} \mathrm{erg} / \mathrm{cm} 34$ & & \\
\hline
\end{tabular}
when $H>2$, the graph of $\gamma$ is a double-well curve, which typically results in the spinodal decomposition of the alloy. The condition $H>2$ is equivalent to $T<T_{c}=\alpha_{i n t} / 2 k \nu$ [30], and for the parameters in Table I] $T_{c}=455 \mathrm{~K}$. Other parameters are: $G=\gamma_{A} / \gamma_{B}$, the ratio of the surface energies of the components; $N=k T \nu / \gamma_{B}$ : the alloy entropy; $\xi=\epsilon / a \gamma_{B}$ : the Cahn-Hilliard gradient energy coefficient; $Q=q_{A} / q_{B}><0$ : the ratio of the effective charges; $F_{e}=q_{B} a \nu V / L \gamma_{B}><0$ : the electric field strength.

TABLE I: Physical and dimensionless parameters for fcc $\mathrm{Ag} / \mathrm{Pt}(111)$ alloy. $a$ is the computed covalent diameter of the Ag atom, which corresponds to a B atom in the model. For the discussion of $\alpha_{i n t}$ see Sec. IV

The dimensionless kinetic transport coefficients are plotted in Fig. 2. Note that at $\Gamma \rightarrow 0$, meaning $\Gamma_{B} \gg \Gamma_{A}$, only $\bar{L}_{B B}$ is not zero, thus $A$ atoms are immobile. And at $\Gamma \rightarrow \infty$, meaning $\Gamma_{A} \gg \Gamma_{B}, \bar{L}_{B B}$ and $\bar{L}_{A B}$ are negligible in comparison to $\bar{L}_{A A}$, thus $\mathrm{B}$ atoms are effectively immobile. At $\Gamma=1\left(\Gamma_{A}=\Gamma_{B}\right), \bar{L}_{A A}=\bar{L}_{B B}$. 


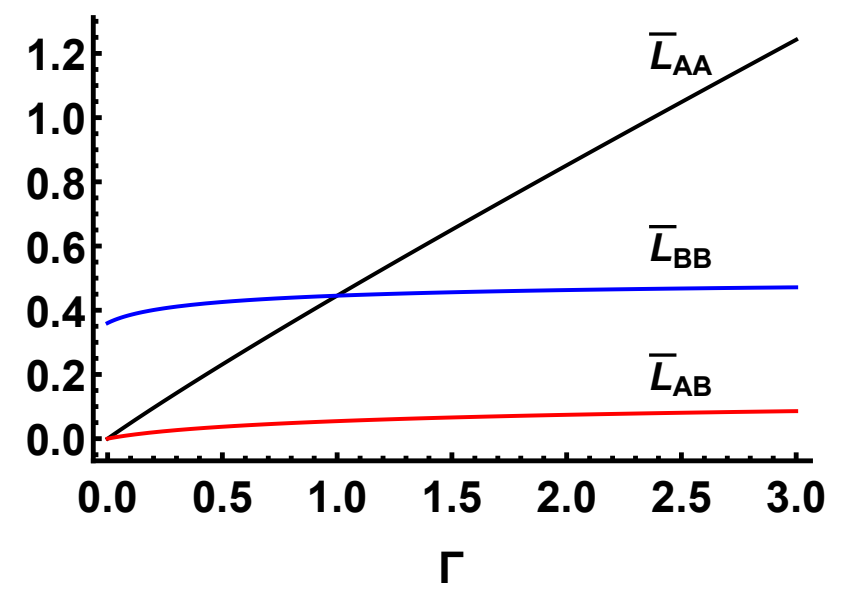

FIG. 2: Kinetic transport coefficients $\bar{L}_{A A}, \bar{L}_{A B}$ and $\bar{L}_{B B}$ at $C_{B}=0.5$. At $\Gamma=2$ (A is fast diffuser), $\bar{L}_{A A}=0.851, \bar{L}_{B B}=0.463$, $\bar{L}_{A B}=0.074$. At $\Gamma=0.2$ (B is fast diffuser), $\bar{L}_{A A}=0.096, \bar{L}_{B B}=0.4, \bar{L}_{A B}=0.02$. These values mildly change as $C_{B}$ is varied, and they are provided only for comparison of the magnitudes, not for computation.

Fig. 3 shows the graphs of the perturbation growth rate $\omega$ from the linear stability analysis (LSA) of Eq. (15) (linearized about $C_{B}=0.5$ ). As calculated, $\omega$ is a function of the perturbation wavenumbers $k_{x}$ and $k_{y}$ in the $x$ and $y$ directions, respectively; here we plotted the cross-section, $\omega\left(k_{x}, 0.2\right)$ for the parameters in Table I. except $V=F_{e}=0$ (no electromigration). Here, due to the symmetry in the absence of anisotropy $(\Lambda=1)$, a graph of $\omega\left(c, k_{y}\right), c=$ const. is the same as a graph of $\omega\left(k_{x}, c\right)$. ( $\omega$ is complex-valued in the presence of electromigration, see for example Ref. 8], but the real part of $\omega$ is not affected by electromigration.) The maximum of the plotted $\omega$ curve shifts toward larger wavelengths as $\Gamma$ decreases. At $\Gamma \leq 0.97$ the entire curve is located below the $k_{x}$ axis for any fixed $k_{y}(\omega<0)$, thus for such $\Gamma$ values any small perturbation of a homogeneous state $\mathrm{A}_{0.5} \mathrm{~B}_{0.5}$ would decay in time, and the homogeneous state would be restored. In other words, phase separation is absent and there is phase stability. Note that this situation occurs at values of the thermodynamic parameters $H, G$ and $N$ that in a Cahn-Hilliard-type model with a generic diffusion account would result in the spinodal instability of the $\mathrm{A}_{0.5} \mathrm{~B}_{0.5}$ alloy; thus at $\Gamma \leq 0.97$ the vacancy-mediated diffusion eliminates the said instability. For this reason, in the next section only $\Gamma>0.97$ is considered. In Section IV we provide the insight into the origin of this interesting and important effect.

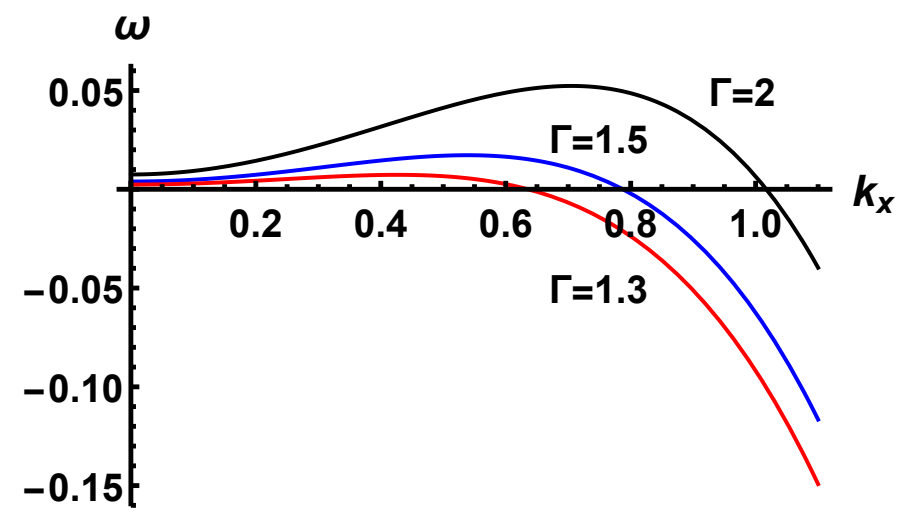

FIG. 3: The perturbation growth rate from LSA of Eq. 15 at $F_{e}=0$.

\section{COMPUTATIONAL RESULTS}

Computations of Eq. (15) are performed on the fixed, square, bi-periodic domain of the size 158a, i.e. 158 lattice spacings, which approximately equals to one-half the width of the terrace $(L / 2 a=152)$. For the parameters in Table [1. 158 lattice spacings equals to $13 \lambda_{\max }$, where $\lambda_{\max }$ is the most dangerous wavelength from LSA. The initial condition $C_{B}(x, y, 0)$ is chosen in the form of a local composition inhomogeneity positioned at the square center; see Fig. 4(a). Within this initial cluster (size: $4 \times 4$ lattice spacings), the concentration of $\mathrm{B}$ atoms is increased to $60 \%$ (see the color bar). This is $10 \%$ higher than outside of the cluster, where the species $\mathrm{A}$ and $\mathrm{B}$ have equal concentrations, that is, $50 \% \mathrm{~A} / 50 \% \mathrm{~B}$. 

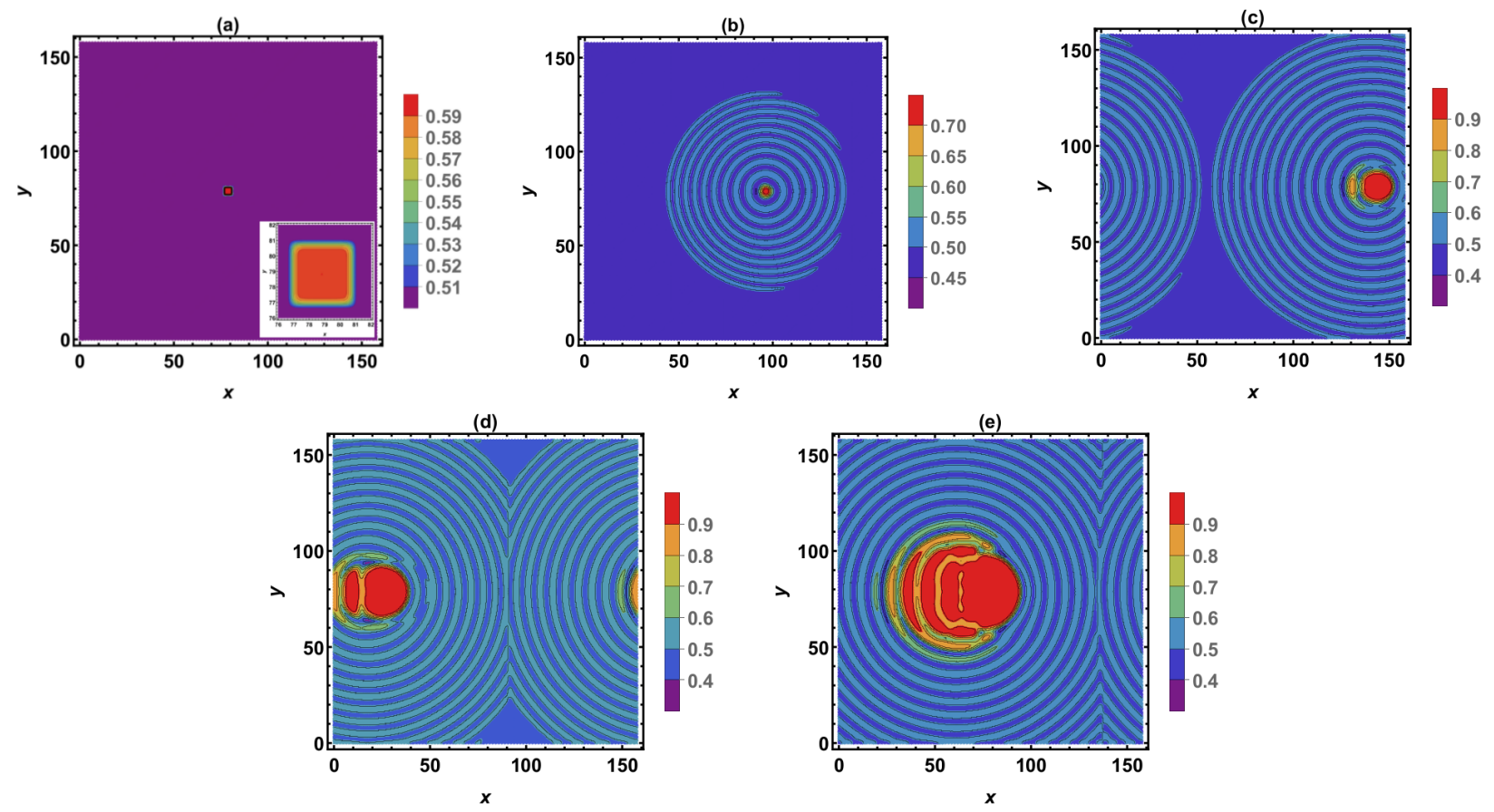

FIG. 4: $C_{B}$ at (a): $t=0$ (the initial condition), (b): $t=20,(\mathrm{c}): t=70,(\mathrm{~d}): t=110,(\mathrm{e}): t=160$. The initial cluster is enlarged in the inset of (a). Values of all parameters are in Table I

Panels (b)-(e) of Fig. 4 show the transport (drift) of the cluster due to the electromigration effect caused by the electric field $\boldsymbol{E}$ in the $180^{\circ}$ direction (see Fig. 1). The dimensionless parameters are taken from Table I. After drifting across the half of the computational domain, the cluster re-emerges at the left boundary of the domain due to the periodic boundary condition. By $t=160$ the center of the cluster covered the distance roughly equal to the domain width of 158 lattice spacings. To obtain a dimensional cluster's speed, first we estimate value of $\Gamma_{B}$ from the Arrhenius-type expression $\Gamma_{B}=\sigma \exp \left(-E_{a} / k T\right)$, where $\sigma=10^{13} \mathrm{~Hz}$ is the attempt frequency and $E_{a} \simeq 1 \mathrm{eV}$ the activation energy [35. [46] This gives $\Gamma_{B}=4 \mathrm{~Hz}$, and then at the parameters values in Table I the time scale $\bar{t}=0.0675 \mathrm{~s}$. Using this value, $t=158$ corresponds to $11 \mathrm{~s}$, and the cluster's speed is 14 lattice spacings $/ \mathrm{s}$. As the cluster drifts, due to developing phase separation instability the B (Ag) atoms from the surrounding surface layer "flow" into the cluster and correspondingly the A (Pt) atoms flow out. Thus the cluster grows with time. In Fig. 4(e), the dimensions of the cluster's core, e.g. the area whose shape resembles a semi-circle, are $29 \times 36$ lattice spacings. Close to the end of the run, the composition is $100 \%$ B phase, see the color bars in Fig. 4(d,e). The development of the instability, which takes place independently of, and alongside with the drift, also results in the repeated formation and breaking of the "neck" at the trailing edge of the cluster. Small clusters that are shedded this way from the cluster's core evolve into B-rich arcs that drift behind the core. Drawing the analogy from biology, the shape of a medium-sized cluster's core, neck, and a small cluster at the end of a neck (Fig. 4(d)) resembles a jellyfish, with the key distinction that the latter actively self-propells in water, whereas the cluster passively drifts in the electron wind. 

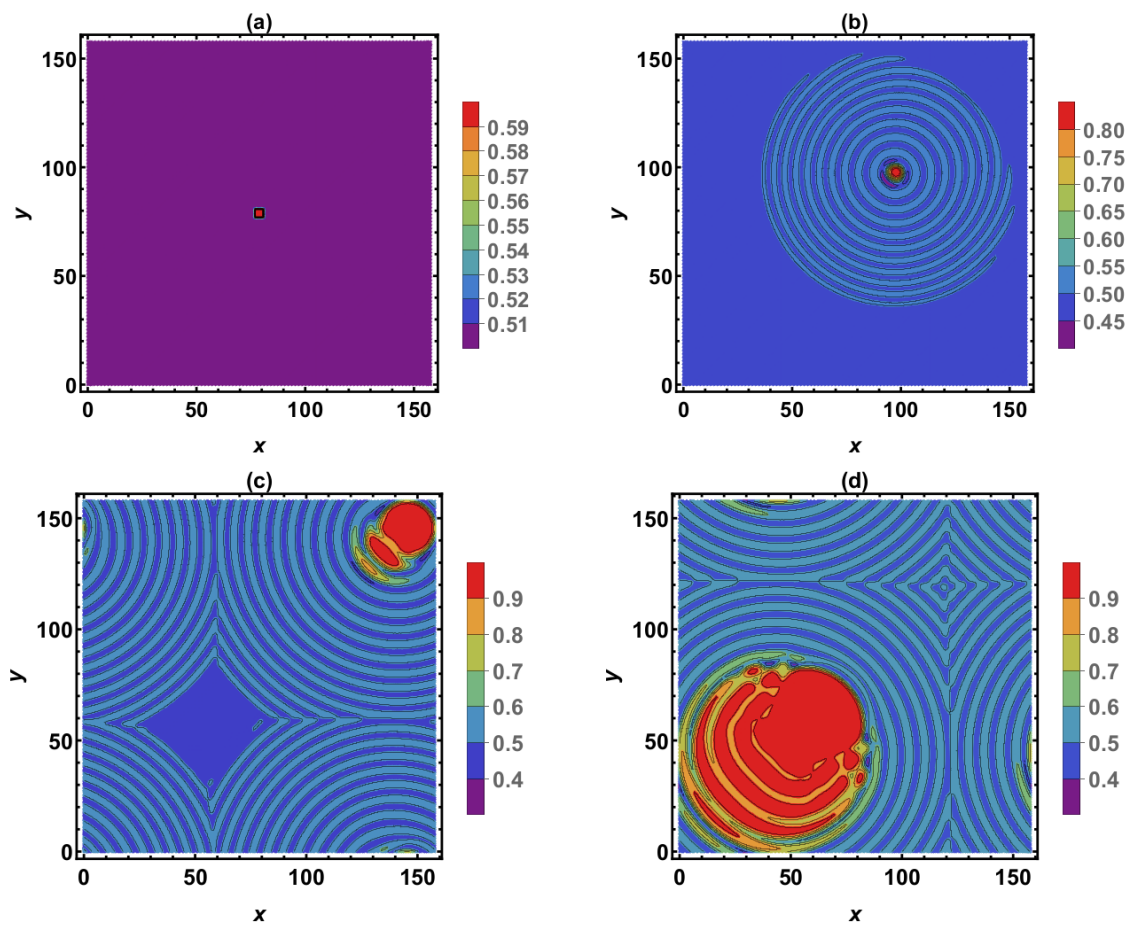

FIG. 5: $C_{B}$ at (a): $t=0$ (the initial condition), (b): $t=30,(\mathrm{c}): t=100,(\mathrm{~d}): t=200 . \phi_{E}=5 \pi / 4$, other parameters as in Table [.

In Fig. 5 the direction of the electric field, and of the current, is $225^{\circ}$, and all other parameters are still as in Table [1. The change in the electic field direction results in the cluster drifting along the square's diagonal with the same speed and other features seen in Fig. 4. From Figures 4 and 5 it is clear that the electromigration very reliably transports the cluster carrying the effective positive charge, across the terrace in the direction that is the opposite to the direction of the current.

In the remaining computations (except the results shown in Figures 8 and 9 we fix the electric field direction to $180^{\circ}$, as in Fig. 4, and study the effects of other parameters.
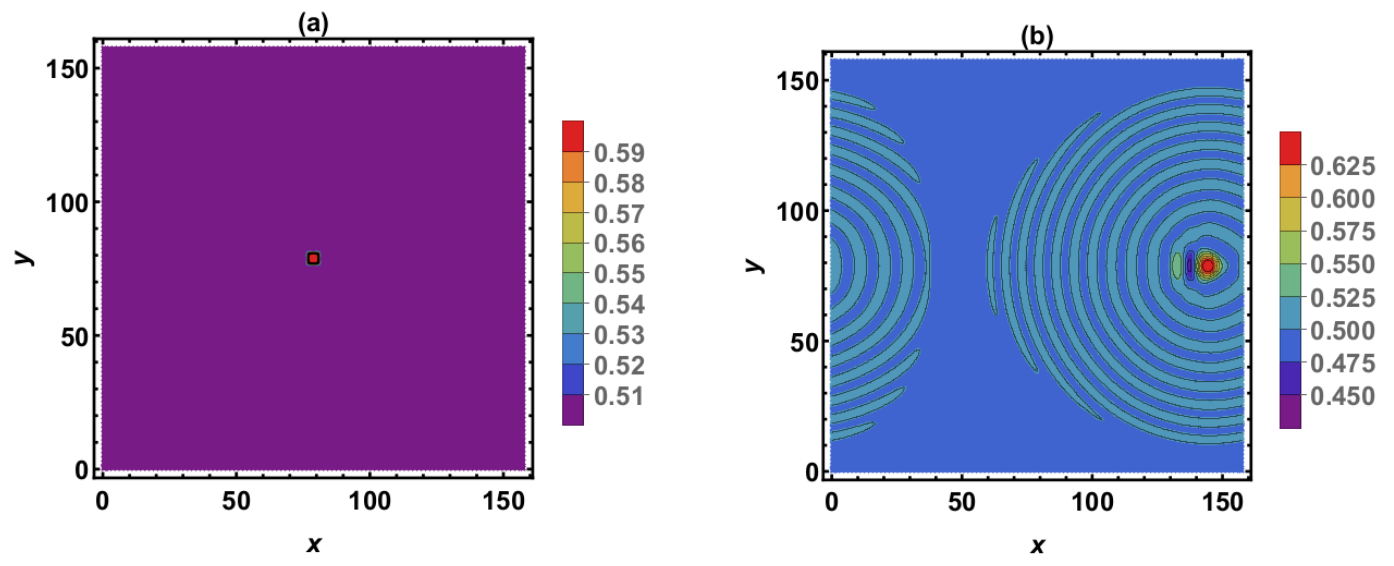

FIG. 6: $C_{B}$ at (a): $t=0$ (the initial condition), (b): $t=100 . \Gamma=1.3$, other parameters as in Table I

Next, Fig. 6 shows the effect of varying the parameter $\Gamma$. As $\Gamma$ is decreased from 2 to 1.3 , the speed of the cluster decreased by over $40 \%$, compare to Fig. 4(c). Since $\Gamma_{B}$ is kept constant (notice that $\Gamma_{B}$ enters only the time scale, which has not changed), the stated decrease of $\Gamma$ is due to the decrease of $\Gamma_{A}$. Thus the slow-down of the drift is attributed to the smaller hop frequency of A atoms, which resulted in the decrease of the kinetic transport coefficients, see Fig. 2 and its caption. Despite the slower cluster drift its enrichment by B atoms is less efficient than at $\Gamma=2$, and the cluster's size and concentration are smaller than in Fig. 4(c). This is consistent with the LSA, see the discussion of Fig. 3 (the phase separation is partially or completely mitigated by decreasing $\Gamma$ ). Computation on this fixed 
domain at $\Gamma<1.3$ still resulted in the cluster drift, but with the phase separation diminishing with time, until the cluster dissolved into the surface.
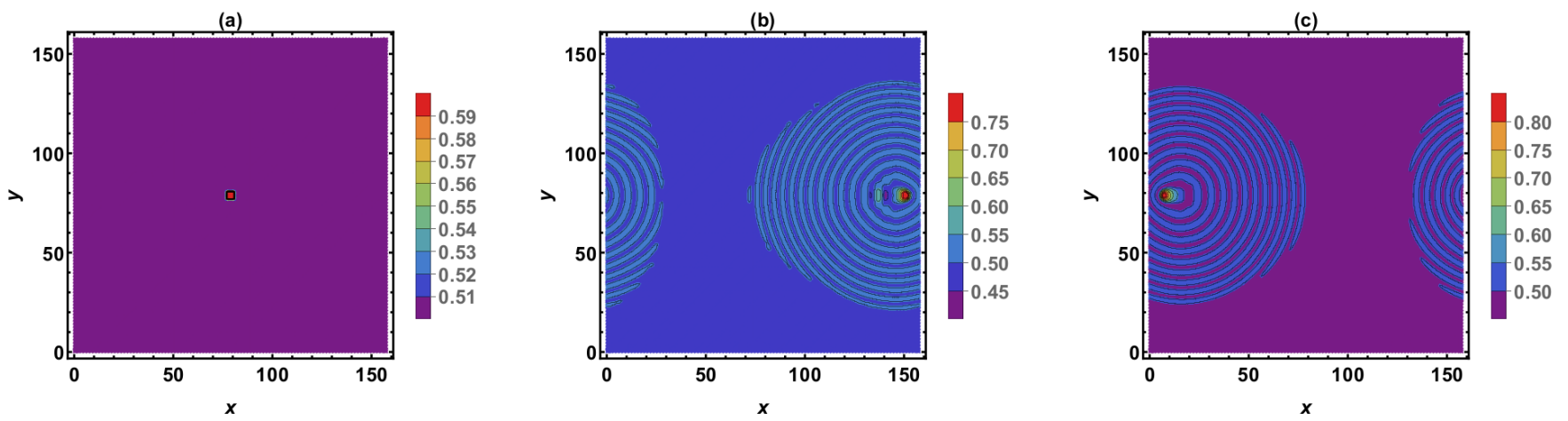

FIG. 7: $C_{B}$ at (a): $t=0$ (the initial condition), (b): $t=70, q_{A}=5 q_{B}>0(Q=5)$, other parameters as in Table $\mathrm{I}$, (c): $t=80$, $q_{A}=-5 q_{B}<0(Q=-5)$, other parameters as in Table $\mathrm{I}$

In Fig. $7(\mathrm{~b})$ it is seen that larger (but still positive) effective charge of A atoms $\left(q_{A} \rightarrow 5 q_{A}\right)$ results in smaller cluster and smaller $C_{B}$ within the cluster, while the drift speed is not affected (compare to Fig. 4(c)). Less efficient phase separation here is the purely kinetic effect of electromigration, since a thermodynamic force that causes phase separation remains constant. To understand the origin of this kinetic effect, observe in Eq. (17) that the parameter $Q$ enters asymmetrically the expressions for electromigration fluxes $\boldsymbol{\Omega}_{A}$ and $\boldsymbol{\Omega}_{B}$. As seen in Fig. 7 (c) and as expected, changing not only the magnitude, but also the sign of $q_{A}$ results in the reversal of the drift direction, with other features of Fig. 7(b) remain essentially unchanged.

\begin{tabular}{|c|c|c|c|}
\hline Applied voltage (V) & Dimensionless speed & Dimensionless size & Composition (\% B) \\
\hline \hline 0.005 & 0.33 & $38 \times 33$ & 95 \\
\hline 0.01 & 0.47 & $26 \times 29$ & 95 \\
\hline 0.015 & 0.63 & $19 \times 23$ & 95 \\
\hline 0.02 & 0.825 & $15 \times 15$ & 95 \\
\hline 0.0225 & 0.94 & $12 \times 11$ & 95 \\
\hline 0.025 & 1.01 & $14 \times 13$ & 95 \\
\hline
\end{tabular}

TABLE II: Cluster speed, size, and composition. The cluster's core size and composition are stated at a time when the cluster reaches the right boundary of the computational domain. Note that values of the applied voltage can be converted into values of the current density using the calculation shown in Sec. IV

Again for the parameters in Table I] Table II shows that the cluster speed increases linearly with the increase of the applied voltage, whereas the cluster size decreases. (The higher the voltage and thus the speed, the less time is afforded to the phase separation instability to replace A atoms on the cluster boundary with B atoms.) The linear fit to speed vs. voltage data is $v=34 V+0.16$; the linear law breaks down at small $V$. We found that at $V=0.0005 \mathrm{~V}$ the cluster is essentially immobile on the time scale $0 \leq t \leq 500$. That is, it stays very close to the center of the computational domain and grows until it fills the entire domain area. 

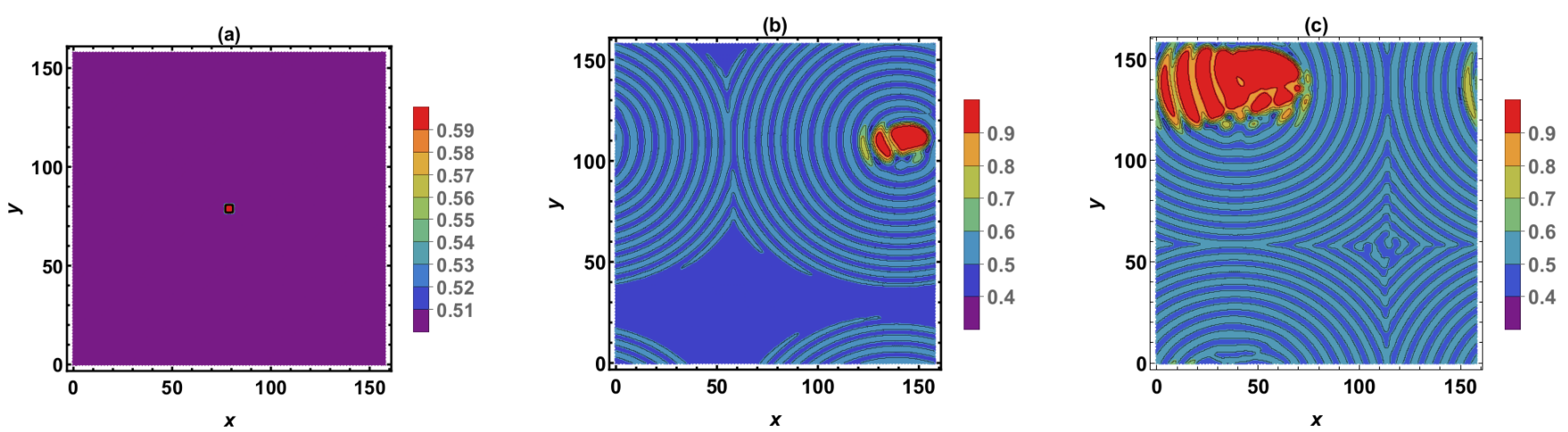

FIG. 8: $C_{B}$ at (a): $t=0$ (the initial condition), (b): $t=10,(\mathrm{c}): t=190 . \Lambda=0.5, \phi_{E}=5 \pi / 4$, other parameters as in Table I
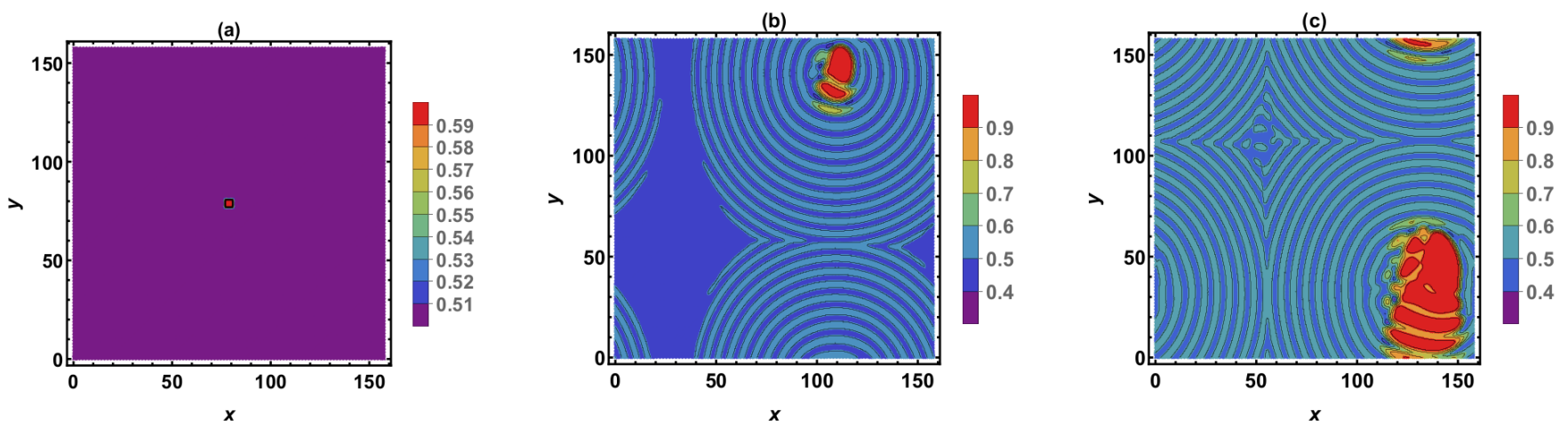

FIG. 9: $C_{B}$ at (a): $t=0$ (the initial condition), (b): $t=50,(\mathrm{c}): t=90 . \Lambda=2, \phi_{E}=5 \pi / 4$, other parameters as in Table $\mathrm{I}$.

Lastly, in Figures 8,9 we clarify the impact of the diffusion anisotropy. The computation is done with $\Lambda=0.5$ and $\Lambda=2$, respectively, using $\phi_{E}=225^{\circ}$. The results should be compared to Fig. 5. It is clear that strong anisotropy has the detrimental effect on the target direction of the drift. [47. At $\Lambda=0.5$ the diffusion in the $x$-direction is twice as strong as in the $y$-direction, which results in the cluster straying, by about $30^{\circ}$ clockwise, from the path along the square's diagonal. At $\Lambda=2$, when the diffusion in the $y$-direction is twice as strong as in the $x$-direction, the deviation from the path along the diagonal is roughly $30^{\circ}$ counter-clockwise. The shape of the cluster is also impacted: losing a near-circular symmetry, the cluster's core is elongated and bended.

\section{DISCUSSION}

We begin this section with estimates of the electromigration current density, the temperature rise of the film, and the enthalpy of mixing of the surface alloy.

Let $\rho$ be the resistivity of $\mathrm{Ag}_{x} \mathrm{Pt}_{1-x}$ film. Then for the electromigration current density $j$ we have:

$$
j=E_{0} / \rho=V /(L \rho), \quad \rho=(1-x) \rho_{P t}+x \rho_{A g},
$$

where $\rho_{A g}=2.24 \times 10^{-6} \Omega \mathrm{cm}, \rho_{P t}=14.7 \times 10^{-6} \Omega \mathrm{cm}$ at $400 \mathrm{~K}$ [36, 37. Substituting these values, $x=0.5$, and $V, L$ from Table I] gives $\rho=8.47 \times 10^{-6} \Omega \mathrm{cm}, j=2.65 \times 10^{8} \mathrm{~V} / \Omega \mathrm{cm}^{2}$.

The temperature rise of a thin metal film on a thick dielectric substrate due to electromigration currrent can be calculated using Black's formula [38]:

$$
\Delta T=\rho j^{2} h_{m f} h_{d s} / \chi_{d s},
$$

where $h_{m f}$ and $h_{d s}$ are the thickness of a film and of a substrate, respectively, and $\chi_{d s}$ is the thermal conductivity of a substrate. Using $h_{m f}=20 \mathrm{~nm}, h_{d s}=200 \mathrm{~nm}, \rho, j$ as calculated above, and $\chi_{d s}=\chi_{P t} \approx 75 \mathrm{~W} / \mathrm{m} \mathrm{K}$ at $400 \mathrm{~K}$ 39, 40] gives $\Delta T=30 \mathrm{~K}$. [48. Thus despite the temperature rise from $400 \mathrm{~K}$ to $430 \mathrm{~K}$ due to electromigration, the film is still below the critical temperature $T_{c} \sim 455 \mathrm{~K}$ of the spinodal decomposition, provided that the chosen value of the enthalpy of mixing $\alpha_{i n t}=0.1 \gamma_{B}$ in Table \is order-of-magnitude accurate. 
To confirm this, we convert $\alpha_{\text {int }}=27 \times 10^{-3} \mathrm{eV} /$ atom for $\mathrm{Ag}_{0.5} \mathrm{Pt}_{0.5}$ alloy [19] to a value in units of erg $/ \mathrm{cm}^{2}$. Using $a / 2$ from Table I for the radius of $\mathrm{Ag}$ atom, and the radius of $\mathrm{Pt}$ atom $1.77 \times 10^{-8} \mathrm{~cm} \mathrm{[27}$, we obtain the average radius $r_{a v}=1.71 \times 10^{-8} \mathrm{~cm}$. Asssuming first that the packing of the surface layer is by solid discs of the radius $r_{a v}$, representing the surface atoms, we obtain $1.1 \times 10^{15}$ for the number of atoms in one square centimeter of the surface, and then $\alpha_{i n t}=47 \mathrm{erg} / \mathrm{cm}^{2}$. Alternatively, assuming that the packing of the surface layer is by solid squares of the side $r_{a v}$, we obtain $3.4 \times 10^{15}$ for the number of atoms in one square centimeter of the surface, and then $\alpha_{i n t}=148$ $\mathrm{erg} / \mathrm{cm}^{2}$. The chosen value $\alpha_{i n t}=117 \mathrm{erg} / \mathrm{cm}^{2}$ in Table I falls neatly between these bounds.

For completeness, in the following remark we compare data from literature on diffusion rates of various atom exchange processes.

For example, according to first principle calculations by Grant et al. 2 for $\mathrm{PdCu}(001)$ surface alloy, the activation energy for $\mathrm{Pd}-\mathrm{Cu}$ adatom exchange is $0.95 \mathrm{eV}$, while the activation energy for vacancy diffusion is only $0.466 \mathrm{eV}$. Here, adatom exchange means that $\mathrm{Pd}$ atom pops out from the surface layer onto the surface, diffuses on the surface, and then reincorporates back into the surface layer. Thus based on these values vacancy-mediated diffusion is expected to predominate. As another example, Anderson et al. 41] found that $\mathrm{Pb}$ atoms embedded in the $\mathrm{Cu}(111)$ surface suppress surface diffusion rates by several orders of magnitude - the surface diffusion barrier for $\mathrm{Cu}$ adatom diffusion increases from $0.8 \mathrm{eV}$ to $0.9 \mathrm{eV}$ with $0.11 \mathrm{ML} \mathrm{Pb}$, and to $1.2 \mathrm{eV}$ with $0.22 \mathrm{ML} \mathrm{Pb}$. This is another sign that vacancy-mediated diffusion is predominant.

To end this section, next we provide the formal explanation of the suppression of the phase separation at the small values of the ratio of the hop frequencies $\Gamma$, as seen in LSA (see section III).

It is sufficient for this purpose to consider the simplified Eq. (15),

$$
\frac{\partial C_{B}}{\partial t}=\left(1-C_{B}\right) \nabla \cdot\left(\bar{L}_{A A} \nabla \mu_{A}\right)-C_{B} \boldsymbol{\nabla} \cdot\left(\bar{L}_{B B} \nabla \mu_{B}\right) .
$$

Here, the electric field parameter $F_{e}=0$, the convective terms are omitted, isotropic diffusion is assumed, and the contributions proportional to $\bar{L}_{A B}$ also are omitted (since it is seen in Fig. 2 that $\bar{L}_{A B} \ll \bar{L}_{A A}, \bar{L}_{B B}$ for any $\Gamma$ ). Also, we can "freeze" the coefficients of two terms on the right-hand side of Eq. 225 by fixing $C_{B}$; we need to take $C_{B}=1 / 2$, since this value is used in LSA and computation for the initial composition. Via the chemical potentials $\mu_{A, B}$ (Eqs. (19)), Eq. 25) contains only the thermodynamic effects, which are affected by vacancy diffusion kinetics via the composition-dependent coefficients $\bar{L}_{A A}$ and $\bar{L}_{B B}$. We now simplify the equation further by assuming that $\bar{L}_{A A}, \bar{L}_{B B}$ are constant; and take $\bar{L}_{A A}=\theta \bar{L}_{B B}, 0<\theta<1$. This choice of the interval for $\theta$ corresponds to the relation between $\bar{L}_{A A}$ and $\bar{L}_{B B}$ in Fig. 2 at $\Gamma<1$. Next, taking $\xi=0$ without loss of generality, and substituting $\mu_{A, B}$ gives

$$
\frac{\partial C_{B}}{\partial t}=\frac{\bar{L}_{B B}}{2} \nabla^{2}\left[C_{B}(1-\theta)-1\right] \frac{\partial \gamma}{\partial C_{B}} .
$$

We again fix $C_{B}$ to a constant in the interval [0,1] (a particular value 1/2 may be chosen), then it is obvious that $C_{B}(1-\theta)-1=-w$, where $w>0$ is another constant. Finally, Eq. 26) reads

$$
\frac{\partial C_{B}}{\partial t}=\frac{-w \bar{L}_{B B}}{2} \nabla^{2} \frac{\partial \gamma}{\partial C_{B}},
$$

where $\gamma$ is given by Eq. 20p. The LSA of Eq. 27) about $C_{B}=1 / 2$ gives $\omega=N w \bar{L}_{B B}(2-H)\left(k_{x}^{2}+k_{y}^{2}\right)$. Thus $\omega<0$ for all wavenumbers at $H>2$ (e.g., all small perturbations of the homogeneous state $C_{B}=1 / 2$ decay). But $H>2$ is the condition of the spinodal instability. This calculation makes it clear that the relation $\bar{L}_{A A}<\bar{L}_{B B}$, which results at $\Gamma<1$, and the particular choice of the alloy composition $\mathrm{A}_{0.5} \mathrm{~B}_{0.5}$, give rise to the the suppression of the phase separation.

\section{SUMMARY}

To summarize, we suggested harnessing a phase separation instability and surface electromigration to enable transport across terraces of A or B atom clusters embedded in the topmost surface layer of a thermodynamically unstable surface binary alloy (e.g., the alloy's enthalpy of mixing is positive). Our synthetic continuum model rests on the prior assessement from STM experiments and ab initio computations that vacancy-mediated diffusion is often predominant for surface alloys. Computations are executed with parameters that closely mimick $\operatorname{AgPt}(111)$ surface alloy, which is known to be thermodynamically unstable and form the embedded Ag clusters. The model is developed by first reducing the classical model of a vacancy-mediated diffusion in the bulk $3 \mathrm{D}$ binary alloy couples to an alloyed, dense 
2D surface layer, and then combining with the consistent, irreversible thermodynamics-based model of electromigration in alloys. The model allows to study the effects that are often overlooked, such as the diffusion anisotropy and the unequal and sign-wise different effective charges of A and B atoms. (To our knowledge, there is no published assessement of the latter effect in any context even for bulk metallic alloys.) For this application, we find that the unequal effective charges may kinetically slow down the phase separation in the binary surface layer. Phase separation also is slowed down or brought to a standstill when the ratio of the hop frequencies of A and B atoms is in certain range. Increasing the applied voltage results in a smaller cluster size and the linear increase of its speed, whereas the purity is not significantly affected. Overall, the model's computations demonstrate the feasibility of accomplishing the stated goal, e.g. enabling the transport across terraces of the embedded A or B atom clusters. Of course, to be successful, a material system chosen for the experimental validation will require carefull optimization and calibration across a multi-parameter landscape.

[1] R. van Gastel, E. Somfai, S.B. van Albada, W. van Saarloos, and J.W.M. Frenken, "Nothing moves a surface: Vacancymediated surface diffusion", Phys. Rev. Lett. 86, 1562 (2001).

[2] M.L. Grant, B.S. Swartzentruber, N.C. Bartelt, and J.B. Hannon, "Diffusion kinetics in the Pd/Cu(001) surface alloy", Phys. Rev. Lett. 86, 4588 (2001).

[3] M.L. Anderson, M.J. D'Amato, P.J. Feibelman, and B.S. Swartzentruber, "Vacancy-mediated and exchange diffusion in a $\mathrm{Pb} / \mathrm{Cu}(111)$ surface alloy: Concurrent diffusion on two length scales", Phys. Rev. Lett. 90, 126102 (2003).

[4] R. van Gastel, E. Somfai, W. van Saarloos, and J.W.M. Frenken, "A giant atomic slide-puzzle", Nature 408, 665 (2000).

[5] H.B. Huntington, Ch. 6: Electromigration in metals, in: Diffusion in Solids: Recent Developments, Ed. A.S. Nowick, Academic Press, 1975.

[6] P.S. Ho and T. Kwok, "Electromigration in metals", Rep. Prog. Phys. 52, 301-348 (1989).

[7] H. Mehrer, Diffusion in solids. Springer (2007).

[8] M. Khenner, "Electromigration-guided composition patterns in thin alloy films: a computational study", Surf. Sci. 698, $121611(2020)$.

[9] S. Curiotto, P. Muller, A. El-Barraj, F. Cheynis, O. Pierre-Louis, and F. Leroy, "2D nanostructure motion on anisotropic surfaces controlled by electromigration", Appl. Surf. Sci. 469, 463-470 (2019).

[10] P. Kuhn, J. Krug, F. Hausser, and A. Voigt, "Complex Shape Evolution of Electromigration-Driven Single-Layer Islands", Phys. Rev. Lett. 94, 166105 (2005).

[11] D. Dasgupta and D. Maroudas, "Surface nanopatterning from current-driven assembly of single-layer epitaxial islands", Appl. Phys. Lett. 103, 181602 (2013).

[12] D. Dasgupta, A. Kumar, and D. Maroudas, "Analysis of current-driven oscillatory dynamics of single-layer homoepitaxial islands on crystalline conducting substrates", Surf. Sci. 669, 25 (2018).

[13] A. Kumar, D. Dasgupta, C. Dimitrakopoulos, and D. Maroudas, "Current-driven nanowire formation on surfaces of crystalline conducting substrates", Appl. Phys. Lett. 108, 193109 (2016).

[14] D. Solenov and K.A. Velizhanin, "Adsorbate transport on graphene by electromigration", Phys. Rev. Lett. 109, 095504 (2012).

[15] C.-H. Chiu, Z. Huang, and C. T. Poh, "Formation of nanoislands by the electromolding self-organization process", Phys. Rev. B 73, 193409 (2006).

[16] J.W. Cahn and J. Hilliard, "Free energy of a nonuniform system. Interfacial free energy", J. Chem. Phys. 28, 258-267 (1958).

[17] J.W. Cahn, "On spinodal decomposition,", Acta Metall. 9, 795-801 (1961).

[18] H. Roder, R. Schuster, H. Brune, and K. Kern, "Monolayer-confined mixing at the Ag-Pt(111) interface", Phys. Rev. Lett. 71, 2086 (1993).

[19] M.H.F. Sluiter, C. Colinet, and A. Pasturel, "Ab initio calculation of the phase stability in Au-Pd and Ag-Pt alloys", Phys. Rev. B 73, 174204 (2006).

[20] S. Zhao, G.M. Stocks, and Y. Zhang, "Defect energetics of concentrated solid-solution alloys from ab initio calculations: $\mathrm{Ni}_{0.5} \mathrm{Co}_{0.5}, \mathrm{Ni}_{0.5} \mathrm{Fe}_{0.5}, \mathrm{Ni}_{0.8} \mathrm{Fe}_{0.2}$ and $\mathrm{Ni}_{0.2} \mathrm{Cr}_{0.2}$ ", Phys. Chem. Chem. Phys. 18, 24056 (2016).

[21] L.S. Darken, "Diffusion, mobility, and their interrelation through free energy in binary metallic systems", Transactions of the Metall. Soc. of AIME 175, 184-201 (1948).

[22] A.M. Gusak, "Nonequilibrium vacancies and diffusion-controlled processes at nanolevel", in: Diffusion-controlled solid state reactions: In Alloys, Thin Films and Nanosystems. Wiley-VCH, 2011.

[23] W.J. Boettinger, J.E. Guyer, C.E. Campbell, and G.B. McFadden, "Computation of the Kirkendall velocity and displacement fields in a one-dimensional binary diffusion couple with a moving interface", Proc. Roy. Soc.: Math., Phys. and Eng. Sci. 463, 3347-3373 (2007).

[24] P.J. Rous, T.L. Einstein, and E.D. Williams, "Theory of surface electromigration on metals: application to selfelectromigration on Cu(111)", Surf. Sci. 315, L995-L1002 (1994).

[25] K.H. Bevan, H. Guo, E.D. Williams, and Z. Zhang, "First-principles quantum transport theory of the enhanced wind force driving electromigration on $\operatorname{Ag}(111)$ ", Phys. Rev. B 81, 235416 (2010). 
[26] Q. Zhang, P.W. Voorhees, and S.H. Davis, "Mechanisms of surface alloy segregation on faceted core-shell nanowire growth", J. Mech. Phys. Solids 100, 21-44 (2017).

[27] E. Clementi, D.L. Raimondi, and W.P. Reinhardt, "Atomic Screening Constants from SCF Functions. II. Atoms with 37 to 86 Electrons", J. Chem. Phys. 47, 1300 (1967).

[28] M. Khenner and V. Henner, "Modeling evolution of composition patterns in a binary surface alloy", Modelling Simul. Mater. Sci. Eng. 29, 015002 (2020).

[29] A.V. Ruban, H.L. Skriver, and J.K. Norskov, "Local equilibrium properties of metallic surface alloys", in: The Chemical Physics of Solid Surfaces, Ed.: D.P. Woodruff, vol. 10, Elsevier (2002).

[30] W. Lu and D. Kim, "Engineering nanophase self-assembly with elastic field", Acta Mater. 53, 3689 (2005).

[31] J.R. Manning, "Correlation factors for diffusion in nondilute alloys", Phys. Rev. B 4, 1111 (1971).

[32] H. Ribera, B.R. Wetton, and T.G. Myers, "Mathematical model for substitutional binary diffusion in solids", arXiv:1911.07359v1 (2019).

[33] L. Vitos, A.V. Ruban, H.L. Skriver, and J. Kolla, "The surface energy of metals", Surf. Sci. 411, 186-202 (1998).

[34] J.J. Hoyt, "Molecular dynamics study of equilibrium concentration profiles and the gradient energy coefficient in $\mathrm{Cu}-\mathrm{Pb}$ nanodroplets", Phys. Rev. B 76, 094102 (2007).

[35] E. Bussmann, I. Ermanoski, P. J. Feibelman, N. C. Bartelt, and G. L. Kellogg, "Buried Pd slows self-diffusion on Cu(001)", Phys. Rev. B 84, 245440 (2011).

[36] R.A. Matula, "Electrical resistivity of copper, gold, palladium, and silver", J. Phys. Chem. Ref. Data 8, 1147 (1979).

[37] J.W. Arblaster, "Selected Electrical Resistivity Values for the Platinum Group of Metals Part I: Palladium and Platinum", Johnson Matthey Technol. Rev. 59, 174-181 (2015).

[38] J.R. Black, "Current limitations of thin film conductors", 20th International Reliability Physics Symposium, San Diego, NV, USA, 300-306 (1982).

[39] Y. Yang, S.M. Sadeghipour, W. Liu, M. Asheghi, and M. Touzelbaev, "Thermal Characterization of the High-ThermalConductivity Dielectrics". In: S.L. Shinde, J.S. Goela (Eds), High Thermal Conductivity Materials. Springer, New York, NY (2006).

[40] Y. Terada, "Thermal Conductivities of Platinum Alloys at High Temperatures", Platinum Metals Rev. 49, 21-26 (2005).

[41] M.L. Anderson, N.C. Bartelt, P.J. Feibelman, B.S. Swartzentruber, and G.L. Kellogg, "The effect of embedded Pb on Cu diffusion on $\mathrm{Pb} / \mathrm{Cu}(111)$ surface alloys", Surf. Sci. 600, 1901 (2006).

[42] O. Pierre-Louis and T.L. Einstein, "Electromigration of single-layer clusters", Phys. Rev. B 62, 697 (2000).

[43] This is supported by ab initio computation for some concentrated fcc alloys. For example, in Ref. 20] it is shown that the formation energies of $\mathrm{Ni}$ and $\mathrm{Co}$ vacancies in $\mathrm{Ni}_{0.5} \mathrm{Co}_{0.5}$ alloy are nearly equal, and they exhibit the same trend as a function of the number of vacancy nearest neighbor pairs.

[44] By a generic diffusion setup we mean a setup like Fick's diffusion equation, e.g. one where the details of diffusion are not considered.

[45] A mean drift velocity term $-F_{e}\left(\boldsymbol{\Omega}_{A}+\boldsymbol{\Omega}_{B}\right) \cdot \boldsymbol{\nabla} C_{B}$ is added to the right-hand side of Eq. 15] prior to computation [42].

[46] Since we could not find the activation energy of vacancy diffusion for AgPt(111) surface alloy, the cited value is for vacancy diffusion on a clean $\mathrm{Cu}$ surface.

[47] Ref. 22 provides the ratio close to 0.25 of the number of the diagonal hops over the distance $\sqrt{2} a$ to the number of hops over the distance $a$ along the $x$ or $y$ direction. This seems to indicate that diffusion anisotropy is typically manifested in vacancy-mediated surface diffusion.

[48] Thermal conductivity of bulk dielectric materials varies greatly, from approximately $1 \mathrm{~W} / \mathrm{m} \mathrm{K}$ for a-SiO$_{2}$ to approximately $2000 \mathrm{~W} / \mathrm{m} \mathrm{K}$ at $400 \mathrm{~K}$ for diamond. 